\title{
Experimental research on thermocapillary migration of drops by using digital holographic interferometry
}

\author{
Shuoting Zhang ${ }^{1} \cdot$ Li Duan $^{1} \cdot$ Qi Kang ${ }^{1}$
}

Received: 23 February 2016 / Revised: 23 May 2016 / Accepted: 24 May 2016 / Published online: 20 June 2016

(C) Springer-Verlag Berlin Heidelberg 2016

\begin{abstract}
The thermocapillary migration of drops in a rectangular cell, with a heated top wall and a cooled bottom wall, was investigated experimentally on the ground. The rectangular test cell was $70 \mathrm{~mm}$ high, with a horizontal cross section of $40 \mathrm{~mm} \times 40 \mathrm{~mm}$. In the present experiment, $30 \mathrm{cSt}$ silicon oil was used as the continuous phase, and a water-ethanol mixture was used as the drop phase, respectively. The drops ranged in size from 1.87 to $6.94 \mathrm{~mm}$ in diameter and were injected into the continuous phase, where the temperature gradients ranged from 0.193 to $0.484{ }^{\circ} \mathrm{C} \mathrm{mm}^{-1}$. In order to measure the temperature distribution of the liquid, a digital holographic interferometry was used, which was non-contact, full-field, and in-situ. The holograms were recorded, and then the corresponding wrapped phase distributions images were numerically reconstructed. The temperature distribution of the continuous phase liquid in the cell had been obtained following the unwrapping. Also, through an algebra layer analysis, the temperature distribution around the drop during the thermocapillary migration was obtained. As a result, the drop was colder than the continuous phase liquid, and a thermal wake existed behind the drop. The influence of convective transport on the drop migration was also investigated for the Marangoni number in the range of 7-174. With the increasing of the Marangoni number, the dimensionless interface temperature difference decreased, which was caused by the convective transport enhanced results in the drop thermocapillary migration velocity becoming decreased. The data were compared with previous space
\end{abstract}

Qi Kang

kq@imech.ac.cn

1 National Microgravity Laboratory, Institute of Mechanics, Chinese Academy of Sciences, Beijing 100190, China experiments to explain the phenomena of the drop migration. Finally, with the increasing Marangoni numbers, the length of the thermal wake region increased, and the thermal wake region became extended.

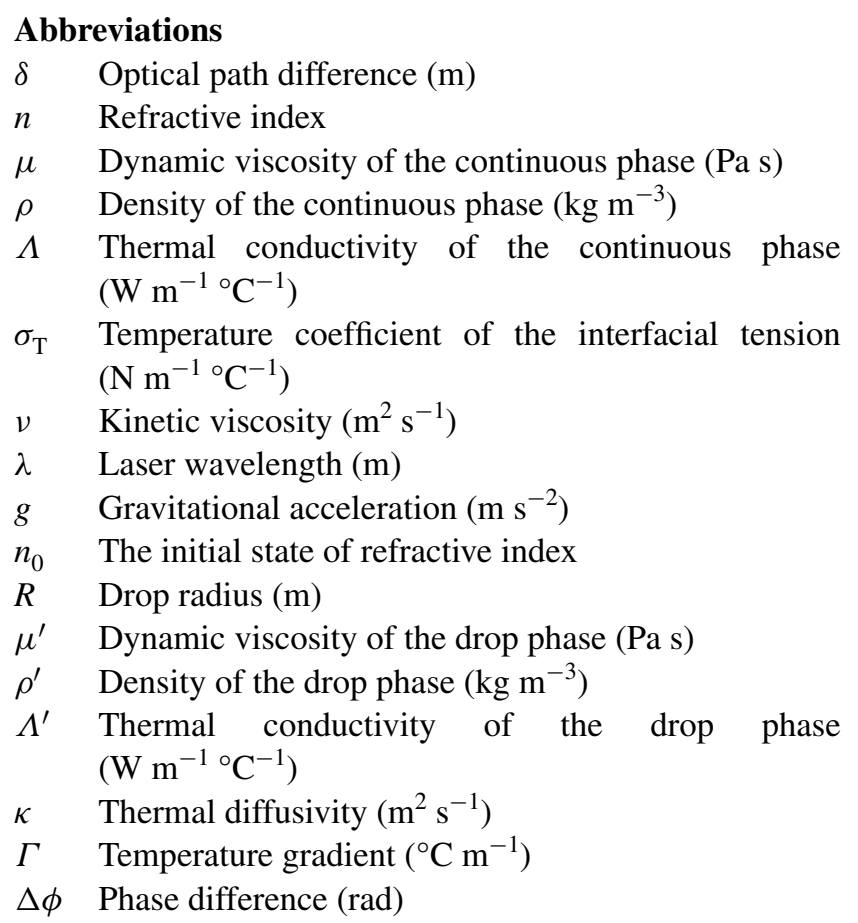

\section{Introduction}

The investigation of thermocapillary flow is important for basic research, as well as for material sciences, chemical engineering, and space manufacturing (Zhu et al. 2011; Zhang et al. 2014). When the gravity effect is greatly reduced, the interfacial phenomenon becomes important. 
The motion of drops driven by temperature gradients is related to the thermocapillary Marangoni convection (Young et al. 1959; Balasubramaniam and Chai 1987). The Marangoni effects are caused by interface tension changes, typically interface tension becoming decreased with increasing temperatures. Under different conditions, The Marangoni effects can cause a drop to move in the opposite direction. When a drop is attached on a non-uniformly heated fibre, temperature variations can be expected to rise at the interface. Then, the local surface stress acts along the surface resulting from the Marangoni effect. The surface stress pulls fluid to the cold side of the fibre. In addition, a friction force acts over the fibre contact surface on the moving drop. Under the pulling force with the friction force acting together, the drop moves to the cold side of the fibre (Yarin et al. 2002). On the other hand, when a drop is placed in a second immiscible fluid in which the temperature gradient has been established, temperature variations can be expected to rise at the interface. The interfacial tension gradient causes the interfacial region of the fluid to generate a pulling force from the lower interfacial tension side to the higher interfacial tension side. This pulling force causes the fluid to move, which generates a tangential stress. The stress transmitted to fluid within the drop cannot cause any force to be exerted on the drop. The stress transmitted to the exterior fluid will cause a reaction force on the same magnitude of the drop, but in the opposite direction. By reaction force, the drop moves to the hot side in the fluid. This mechanism is known as thermocapillary migration (Subramanian and Balasubramaniam 2001). The present study focuses on thermocapillary migration.

Thermocapillary migration has been investigated experimentally on the ground. Young et al. (1959) were the first to investigate the thermocapillary migration of bubbles in liquids, both theoretically and experimentally on the ground. They held a bubble stationary in a temperature gradient and designed a theoretical linear prediction model for the migration velocity of the bubble with a neglecting convective term. After the pioneering work of Young et al., the thermocapillary migration of drops in liquids was investigated on the ground (Rashidnia and Balasubramaniam 1991; Hähnel et al. 1989; Xie et al. 1996). Rashidnia and Balasubramaniam (1991) chose $5 \mathrm{cSt}$ silicon oil and vegetable oil as the continuous phase and drop phase, respectively, in order to provide a simulation of the microgravity condition using a density match method. They observed the velocities of the drops were less than that predicted by a linear theory. Hähnel et al. (1989) investigated the thermocapillary migration of single water drops in butyl benzoate. In this investigation, a systematic deviation from the YGB linear theory was observed, where the convective transport inside and outside the drop had been ignored by the linear theory. Rashidnia and Balasubramaniam (1991) and Xie et al. (1996) observed the flow patterns inside the drop by a long-time exposure and PIV method, respectively. The flow pattern was found to resemble Hill's spherical vortex.

Some microgravity experiments for the thermocapillary migration of bubbles and drops in a sounding rocket and satellite have been performed. These experiments were conducted by Wozniak (1991) to investigate the thermocapillary migration of drops under reduced gravity. The researchers observed that the drop velocity increased with the drop size, as the YGB linear theory had predicted. However, the YGB linear theory predicted that the velocity of the drop would be larger than the ones which were measured during the experiment. An experiment regarding the thermocapillary migration of bubbles was also performed by Kang et al. (2008). These results indicated that the scaled velocity of the bubbles decreased when the increase in the Marangoni number extended to 9288. Among the experimental results mentioned above, the temperature distribution of the continuous phase liquid had not been studied, or the perturbed temperature distribution around and on the interface of the drop during the thermocapillary migration. Also, the coupling between temperature field and thermocapillary migration of a drop have not yet been examined. As these are important factors which will advance the understanding of thermocapillary migration, they are the focus of this present study.

A numerical simulation of thermocapillary drop migration was published by Ma et al. (1999). It was observed in this research that when the convective transport was predominant, the internal circulation led to a new state of motion for the drop. The drop was quite cold when compared to the undisturbed continuous phase liquid, and a long thermal wake existed behind the drop. A topological structure of the temperature fields in deformable drop thermocapillary migration was published by Zhao et al. (2011).

The thermocapillary bubble migration theory was analysed theoretically by Balasubramaniam and Subramanian (1996), and the inner and outer temperature fields of the bubble were obtained. Then, Balasubramaniam and Subramanian (2000) employed a method of matched asymptotic expansions to solve the conjugate heat transfer problem in the thermocapillary migration of a drop. The results indicated that a large temperature difference existed between the surface of the drop and its interior. Also, the variation in temperature over the drop's surface was large. Zhang et al. (2001) observed that the inclusion of inertia was crucial, under the combined action of gravity and thermocapillarity at a small Peclet number, by utilizing an asymptotic method in the thermocapillary migration of a drop. Under the assumptions of a quasi-steady state, along with the non-deformation of a drop, the inner and outer temperature fields of a drop were obtained by Wu (2014). 
Thermal wakes also appear in a liquid which has a temperature gradient. An asymptotic analysis of unsteady-state heat and mass transfer of a droplet was carried out by Polyanin (1984). The interaction of the internal wake was studied. The perturbed temperature field of a drop was studied by Polyanin (1982). A complete asymptotic expansion of a solution in the wake region to the rear of a droplet was constructed by Akhmetov (2004). The thermal wake interaction of two drops with the combined effects of gravity and thermocapillary was examined by Lavrenteva and Nir (2003) and Frolovskaya et al. (2006).

The drop migration in a vertical temperature gradient has been investigated experimentally on the ground. The aim of the present work was to observe the thermocapillary migration of a drop, and to provide quantitative temperature distribution data. To achieve this goal, digital holographic interferometry was used to measure the temperature field. The advantage of the present experimental study was that the digital holographic interferometry was used to measure the temperature field, which was a non-contact, nondestructive, in-situ, and full-field measurement. The temperature distribution in the vertical temperature gradient of the interface, and the surrounding of a drop during its migration, were visually studied and quantitatively provided for the first time. Also, the results of perturbed temperature and thermal wake region are discussed in detail.

\section{Experimental set-up and procedure}

\subsection{Experimental apparatus}

As shown in Fig. 1, a rectangular cell was filled with the test fluid. The top and bottom walls were two aluminium plates, and the four-sided wall was 8.0-mm-thick optical glass. The rectangular test cell was $70 \mathrm{~mm}$ high, with a horizontal cross section measuring $40 \mathrm{~mm} \times 40 \mathrm{~mm}$. A piece of electric heating film and a Peltier element were placed at the top and the bottom surface, respectively, in order to maintain the temperature gradient. Two thermocouples were fixed into the top and bottom walls, and then connected with EUROTHERM 3504 controllers, in order to control the temperature differences between the two walls.

Using the advantages of non-contact, non-destructive, in-situ, and full-field measurements, digital holography was applied in the present experiment for measuring the temperature distribution of the liquid (Duan et al. 2008; Wang et al. 2014). The digital holographic interferometry system was based on a double-exposure holographic interferometry method. Digital holography is a new imaging technique which uses a charge coupled device (CCD) camera for hologram recording, and a numerical method for hologram reconstruction. Furthermore, digital holographic tomography is an effective method for the quantitative measurement of the distributions of temperature fields. In this study, the digital holographic interferometry system was based on the previous research of Northwestern Polytechnical University and was completed in cooperation with the Institute of Mechanics, Chinese Academy of Sciences, as shown in Fig. 2.

As shown in Fig. 3, a laser beam was split into two parts using an optical fibre coupler. Then, both beams were expanded, filtered, and collimated via a beam expander collimation optical system in order to generate the reference and object waves, respectively. The temperature change of the liquid field resulted in the corresponding change of the refractive index of the liquid, thereby causing the phase distribution change of the object wavefront. The object wave passed through the liquid field, and then interfered with the reference wave through another beam splitter. Before the drop injected a digital hologram carrying the information of the liquid field distributions in the initial state, it was
Fig. 1 Schematic diagram of the experimental apparatus

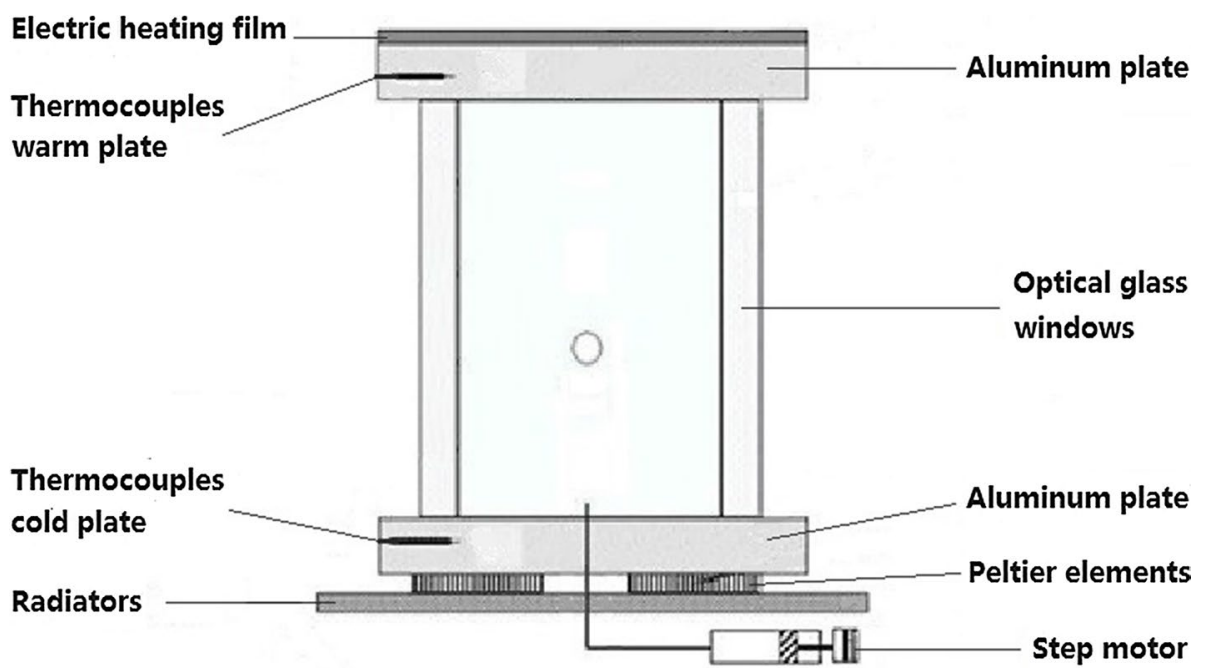


Fig. 2 Experimental apparatus

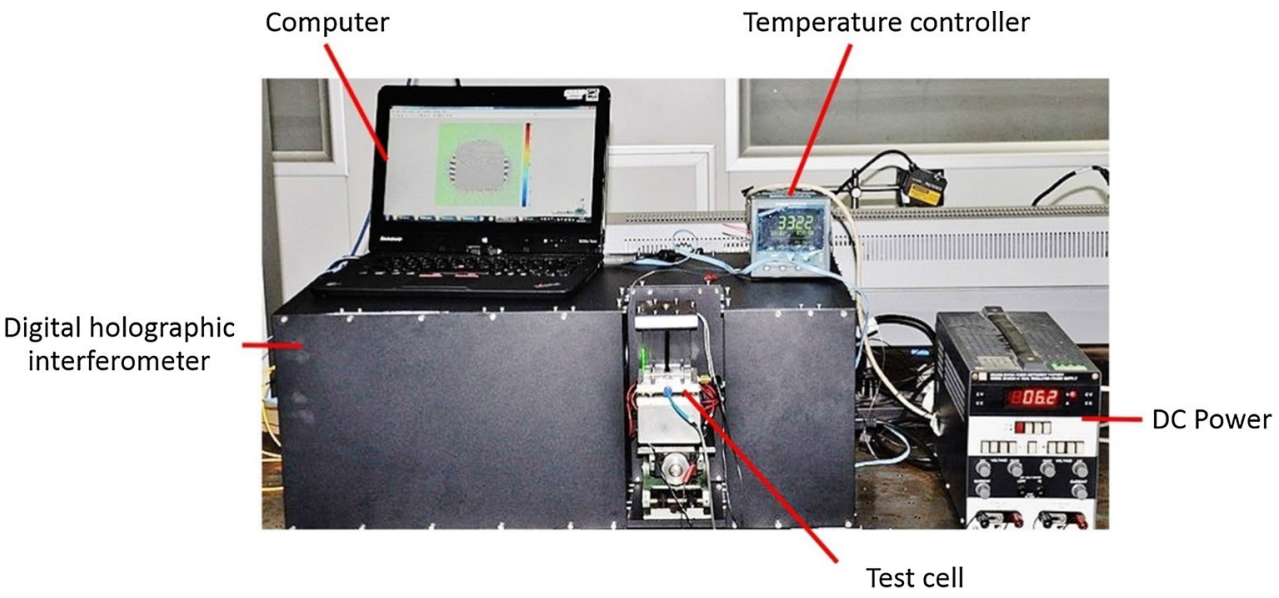

Fig. 3 Experimental set-up for measuring the temperature based on digital holographic interferometry

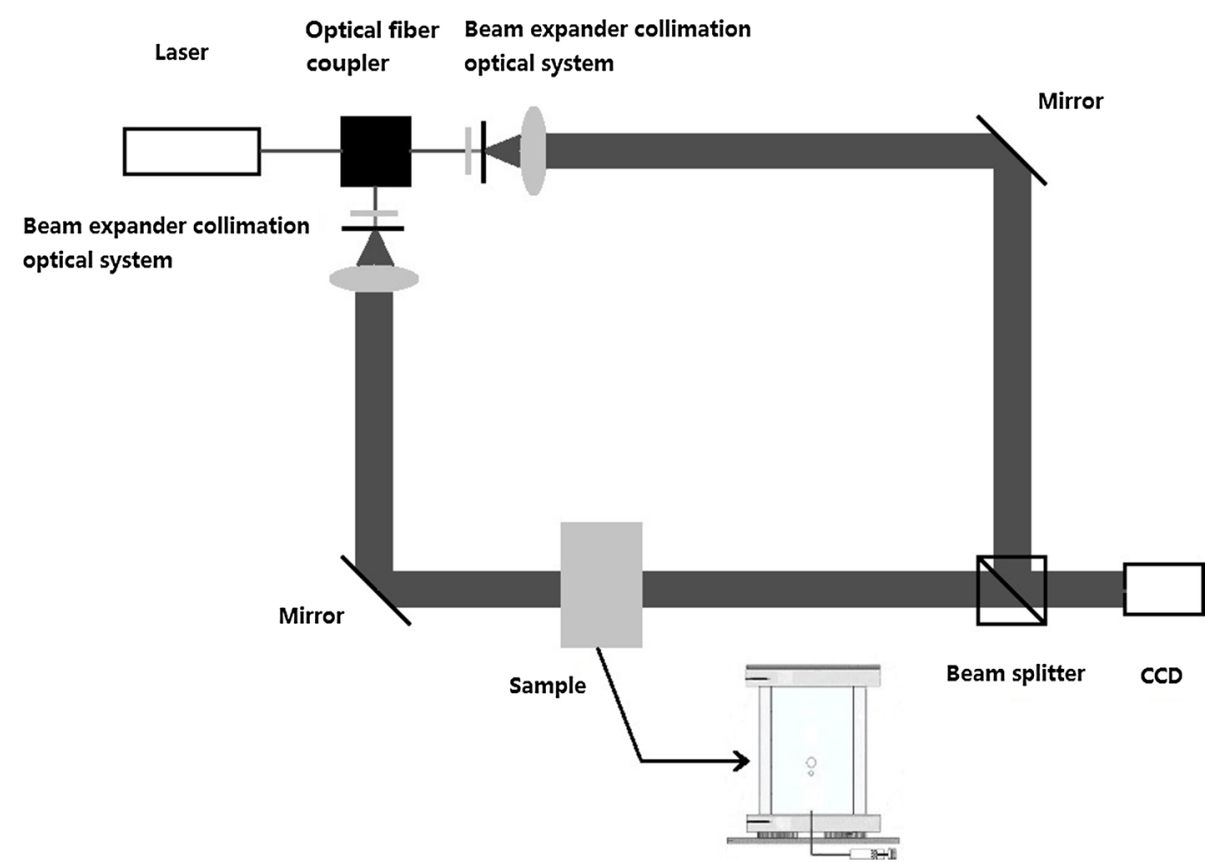

recorded by a CCD camera, and then the drop was injected into the test cell. During the drop thermocapillary migration, a series of digital holograms carrying the information of the liquid field distributions in different states were recorded by the CCD camera. The digital hologram in the initial state and a digital hologram with the drop formed a holographic interferogram which recorded the information of the refraction index field. Then, the corresponding wrapped phase distributions images were numerically reconstructed from the holographic interferograms via a computer. Finally, the corresponding optical path difference of the object wave through the liquid field was calculated.

\subsection{Test liquids}

A KF-96L series silicon oil of nominal viscosity $30 \mathrm{cSt}$, which was made by Shin-Etsu Chemical Co., Ltd. was selected as the continuous phase. This type of dimethyl silicon oil is an ideal experimental material, due to its steady surface tension for contamination. In this study, a water-ethanol mixture was chosen (73-27 by weight) as the drop phase. The silicon oil and water-ethanol mixture were immiscible with approximate densities. The physical property parameters of the liquid system used in the experiment are listed in Table 1. Due to the fact that the temperature difference reached up to $20{ }^{\circ} \mathrm{C}$ in the experimental area, the data of the density variances with temperature are displayed in Table 2.

\subsection{Experimental procedure}

The cell was assembled, and filled with $30 \mathrm{cSt}$ silicone oil. Then, the vertical temperature gradient was established once the desired temperature was obtained in the cell. Two T-type thermocouples were fixed in the upper and 
Table 1 Physical property parameters of the two liquids used for the experiments at $25{ }^{\circ} \mathrm{C}$

Table 2 Densities $\left(\mathrm{kg} \mathrm{m}^{-3}\right)$ at various temperatures

\begin{tabular}{lllll}
\hline Fluid & $v\left(\mathrm{~m}^{2} \mathrm{~s}^{-1}\right)$ & $\rho\left(\mathrm{kg} \mathrm{m}^{-3}\right)$ & $\Lambda\left(\mathrm{W} \mathrm{m}^{-1}{ }^{\circ} \mathrm{C}^{-1}\right)$ & $\sigma_{\mathrm{T}}\left(\mathrm{N} \mathrm{m}^{-1}{ }^{\circ} \mathrm{C}^{-1}\right)$ \\
\hline $30 \mathrm{CS}$ & $3.0 \mathrm{e}-5$ & 955.0 & 0.151 & $-86.3 \mathrm{e}-6$ \\
Water-ethanol mixture & $2.8 \mathrm{e}-6$ & 955.0 & 0.41 & \\
\hline
\end{tabular}

\begin{tabular}{lcccccc}
\hline Fluid & $15{ }^{\circ} \mathrm{C}$ & $20{ }^{\circ} \mathrm{C}$ & $25^{\circ} \mathrm{C}$ & $30{ }^{\circ} \mathrm{C}$ & $35{ }^{\circ} \mathrm{C}$ & $40{ }^{\circ} \mathrm{C}$ \\
\hline $30 \mathrm{CS}$ & 963.3 & 959.4 & 955.0 & 950.6 & 946.4 & 942.2 \\
Water-ethanol mixture & 961.2 & 958.1 & 955.0 & 951.9 & 948.9 & 945.8 \\
\hline
\end{tabular}

lower boundaries of the cell in order to measure the temperature. Then, the electrical source controlled by a temperature controller began to control the electric heating film, as well as the Peltier element working to the desired temperatures. The vertical temperature gradient of the continuous phase liquid was established in the cell. After $2 \mathrm{~h}$, when the steady-state conditions were achieved, the drops were injected into the cell by a step motor. A new drop was inserted $30 \mathrm{~min}$ later, when the perturbation had decayed.

To obtain the perturbed temperature distribution during the entire drop migration process, a digital holographic interferometry system was used. Due to the liquid field which existed in the temperature gradient, the refractive index had a non-uniform distribution. A CCD camera recorded the holograms of the liquid field in the initial and temperature variation states, respectively. By comparing the reconstructed complex amplitude of the object wave in the different states, the change of the optical path of the object wave was obtained. Then, the refractive index information of the liquid field was deduced from the quantitative phase information of the holograms.

Prior to the drop injection, an object wave passed through the liquid field in the initial state without the drop becoming perturbed. The hologram in the initial state recorded the information of the refraction index field $n_{0}(x, y)$. After the drop was injected into the test cell, the object wave passed through the liquid field in a perturbed state, with the drop becoming perturbed. The hologram in the perturbed state recorded the information of the refraction index field $n(x, y)$. As shown in Fig. 4, $Z$ was the symmetry axis of fluid field, during the drop thermocapillary migration, the distribution of the refractive index change $\Delta n\left(\Delta n(r)=\left[n(x, y)-n_{0}(x, y)\right]\right)$ was axisymmetric. The refractive index change distribution was only a function of the radius. The distance between the light and the $x$-axis was $y$, and the light was parallel to the $x$-axis. Therefore, when the light propagated along the $x$ direction, the corresponding optical path difference of the object wave through the liquid field caused by the refractive index change can be expressed as follows:

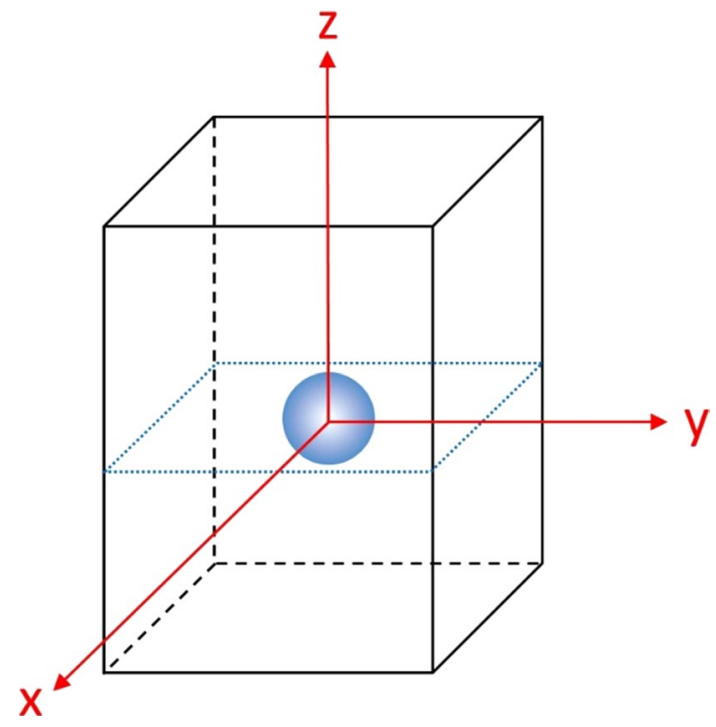

Fig. 4 Schematic diagram of the fluid field

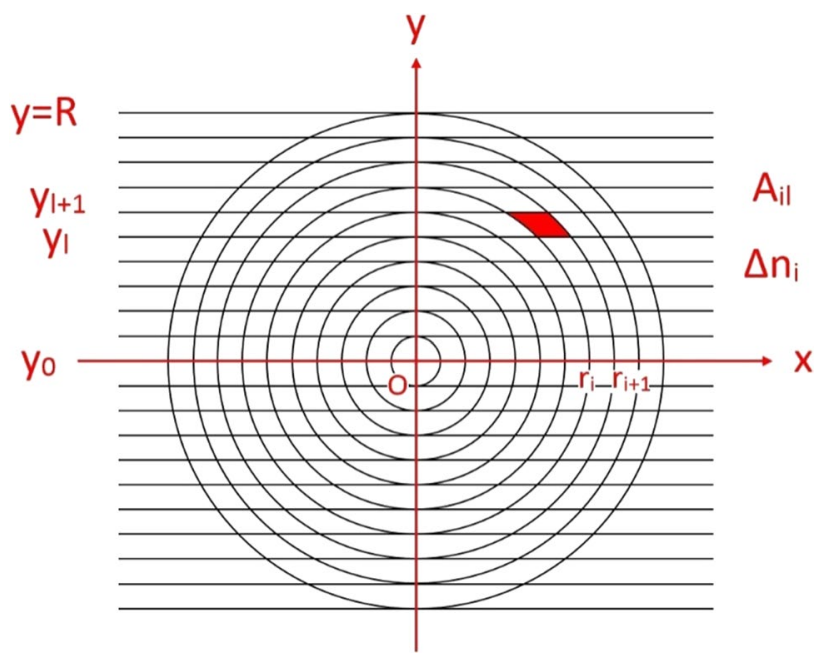

Fig. 5 Region division of the axisymmetric field refractive index change distribution 
$\delta(y)=\int_{-x_{0}}^{x_{0}}\left[n(x, y)-n_{0}(x, y)\right] \mathrm{d} x$

The phase difference can be expressed as:

$\Delta \phi=\frac{2 \pi}{\lambda} \delta$

Then, Eq. (1) was converted into cylindrical coordinates, and expressed as follows:

$\delta(y)=2 \int_{y}^{r_{0}} \frac{\Delta n(r)}{\sqrt{r^{2}-y^{2}}} r \mathrm{~d} r$

As shown in Fig. 5, the axisymmetrical field was divided into k equally spaced concentric annular regions. The light was divided into a bar region. Then, the change of the refractive index was an approximate constant in the bar region. The following summation equation can be used to replace the integral equation:

$\delta_{1}=2 \sum_{i=1}^{k-1} \Delta n_{i}(r) \int_{r_{i}}^{r_{i+1}} \frac{r \mathrm{~d} r}{\sqrt{r^{2}-r_{1}^{2}}}$

$\delta_{1}=2 \Delta r \sum_{i=1}^{k-1} A_{i l} \Delta n_{i}(r)$

The refractive index change distribution could be calculated in turn from the outer to centre, and obtained in the annular region.

In the actual drop thermocapillary migration experiments, the changes of the refractive index and temperature resulting from the drop thermocapillary migration were axisymmetrical. Therefore, using an algebra layer analysis of the reconstruction of the refraction index in the axisymmetrical field, the perturbed temperature distribution and the actual temperature distribution in the continuous phase were obtained.

\section{Experimental results and analysis}

In the current experiment, a digital holographic interferometry system was used to obtain the vertical temperature distribution of the continuous phase liquid in the cell. Then, the holograms were separately recorded using CCD. The corresponding phase distributions of the reconstructing of the object waves were obtained by numerically reconstructing the object waves from the holograms in the initial and steady states. The wrapped phase maps representing the distribution of the temperature field were obtained by subtracting the phase distribution of the initial state from those of the steady states.
After $2 \mathrm{~h}$, when the steady-state conditions were achieved, the hologram was recorded, and the wrapped phase map was numerically reconstructed. After unwrapping the phase map, the temperature distribution of the continuous phase liquid in the experimental area was obtained. The corresponding height of the temperature was calculated in the experimental area. The temperature gradient was observed to be nearly linear along the height of the experimental area.

In regards to the thermocapillary flow, the relative strength of the inertial forces to the viscous forces was measured by the dimensionless Reynolds number $R e$, defined as follows:

$R e=\frac{\left|\sigma_{T}\right| \Gamma R^{2}}{\rho v^{2}}$

The dimensionless Marangoni number $M a$ could then describe the ratio of the convective transport to the thermal conduction in the thermocapillary flow, defined as follows:

$M a=\frac{\left|\sigma_{T}\right| \Gamma R^{2}}{\rho \nu \kappa}$

The dimensionless Capillary number $\mathrm{Ca}$ could then describe the ratio of the surface tension gradient to the surface tension, defined as follows:

$C a=\frac{\left|\sigma_{T}\right| \Gamma R}{\sigma}$

The experimental data were compared with the YGB linear theory, which was used as a guideline. The YGB linear theory migration velocity of the drops was given by:

$V_{\mathrm{YGB}}=\frac{2\left|\sigma_{T}\right| R \Lambda \Gamma}{\left(2 \mu+3 \mu^{\prime}\right)\left(2 \Lambda+\Lambda^{\prime}\right)}+\frac{2\left(\rho-\rho^{\prime}\right) g R^{2}\left(\mu+\mu^{\prime}\right)}{3 \mu\left(2 \mu+3 \mu^{\prime}\right)}$

After the temperature field steady-state conditions were achieved, a drop of $3.70 \mathrm{~mm}$ in diameter was injected into the cell, whose Reynolds number was 0.166, Marangoni number was 50.5, and Capillary number was 0.00270 . The temperature at the different locations was calculated from the drop trajectories. The comparison of the drop velocities with the YGB linear theory is depicted in Fig. 6. It can be seen that the velocity at the beginning was as high as $0.528 \mathrm{~mm} \mathrm{~s}^{-1}$. However, the velocity reached $0 \mathrm{~mm} \mathrm{~s}^{-1}$ at the end of the process. Furthermore, the YGB linear theory velocity was different from the experimental data. The drop migration velocity of the present experiment was found to be less than that of the YGB linear theory. This result was consistent with the measurements obtained in previous research studies (Hähnel et al. 1989). The interfacial tension gradient drove the drop thermocapillary migration. Then, the larger interface temperature gradient resulted 


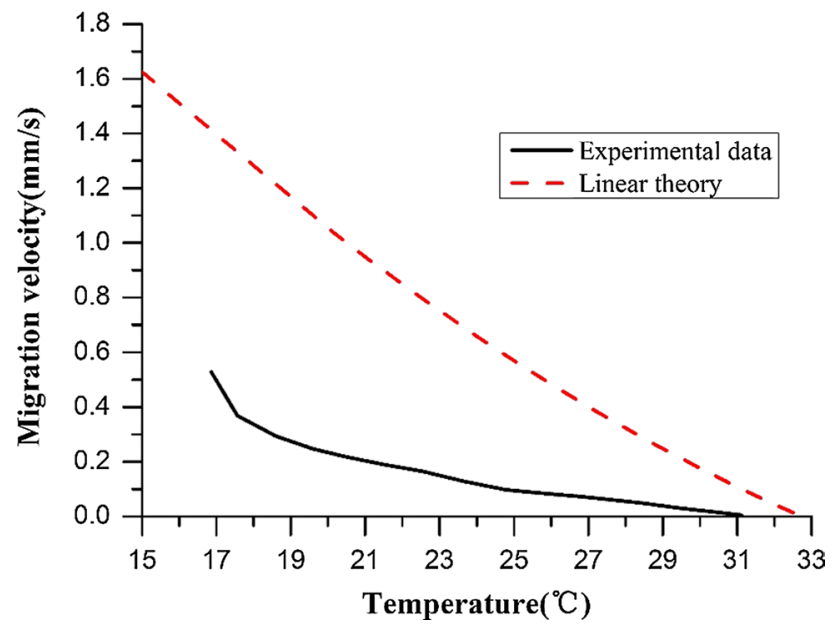

Fig. 6 Comparison of the present experimental data with the linear theory predictions

in a greater driving force, and a faster migration velocity. The YGB linear theory applied to a small Marangoni number condition. However, in the present experiment, the Marangoni number was not negligible. With a high Marangoni number, the interface temperature gradient varied, which was caused by the convective transport enhanced results in drop thermocapillary migration velocity becoming decreased. To validate this concept, it was necessary to measure the temperature distribution around the drop during the experiment. The temperature distribution will be discussed later in this study.
It should be noted that in regards to the drop migration, the experimental investigation of the thermocapillary drop migration was performed on the ground in the current study. The drop migration was induced by the combined effects of buoyancy and thermocapillary forces. However, the liquids had equal densities at $25{ }^{\circ} \mathrm{C}$ and thereby provided a simulation of low-gravity conditions by reducing the buoyancy forces. It can be seen from Fig. 6 that the velocity included the combined effects of buoyancy and thermocapillary forces. In regards to the $3.70 \mathrm{~mm}$ in diameter drop, the absolute value of the second term in Eq. (9) was less than the absolute value of the first term from 19.4 to $32.7^{\circ} \mathrm{C}$.

The drop migration was observed through a digital holographic interferometry system. The holograms were separately recorded via CCD after a time interval of $1 \mathrm{~s}$. Figure 7 shows a sequence of holograms at 0, 20, 40, 60, 80 , and $100 \mathrm{~s}$, respectively. By numerically reconstructing the object waves from the holograms at the temperature field steady state, and when the state of the drop was perturbed by the temperature fields in the continuous phase, the corresponding phase distributions of the reconstructing of the object waves were obtained. Figure 8 shows a sequence of the wrapped phase maps at 0, 20, 40, 60, 80, and $100 \mathrm{~s}$, respectively. Using an algebra layer analysis of the reconstruction of the refraction index in axisymmetrical field, the perturbed temperature distribution in the continuous phase was obtained. The perturbed temperature was defined as the difference between the terminal and initial temperatures at the same position. Figure 9 shows
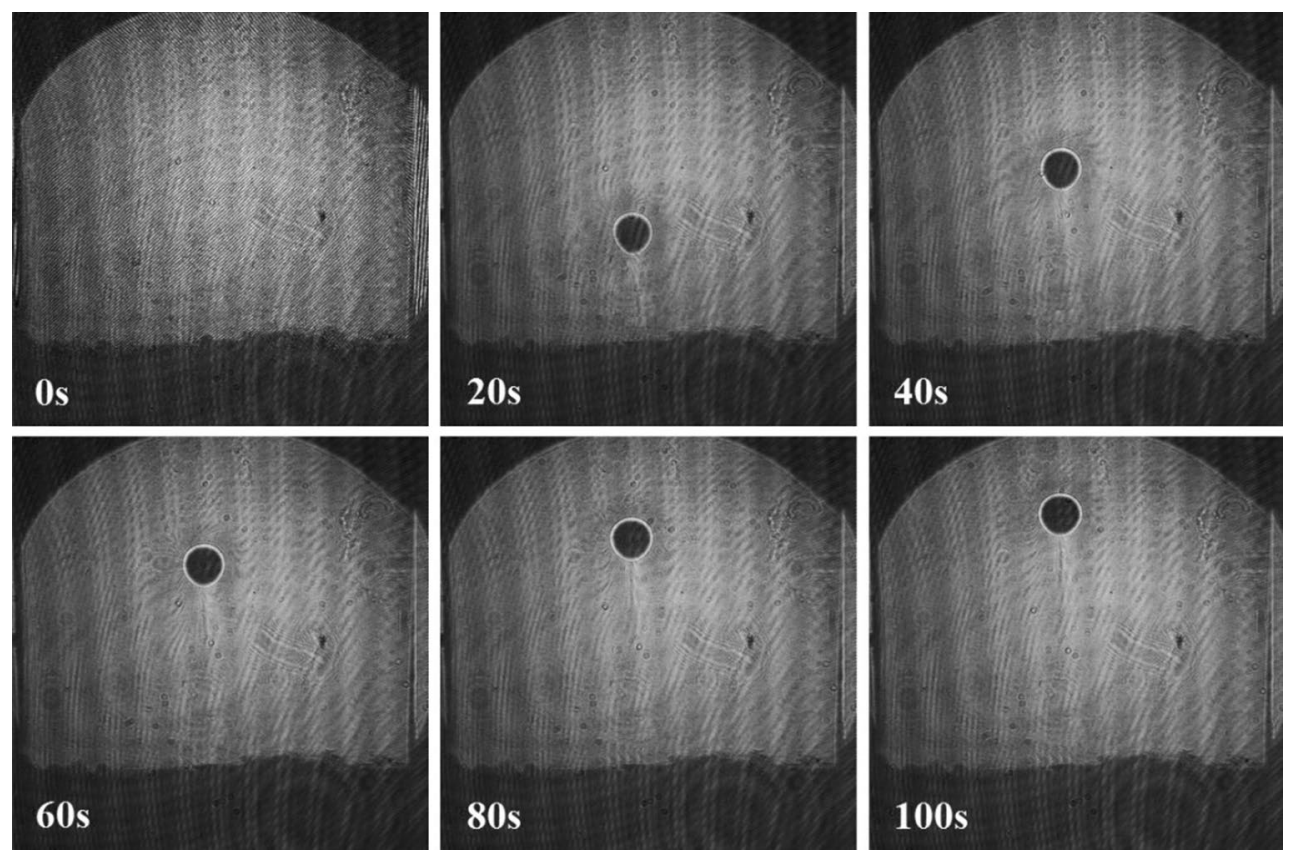

Fig. 7 Holograms of the drop migration at different times 

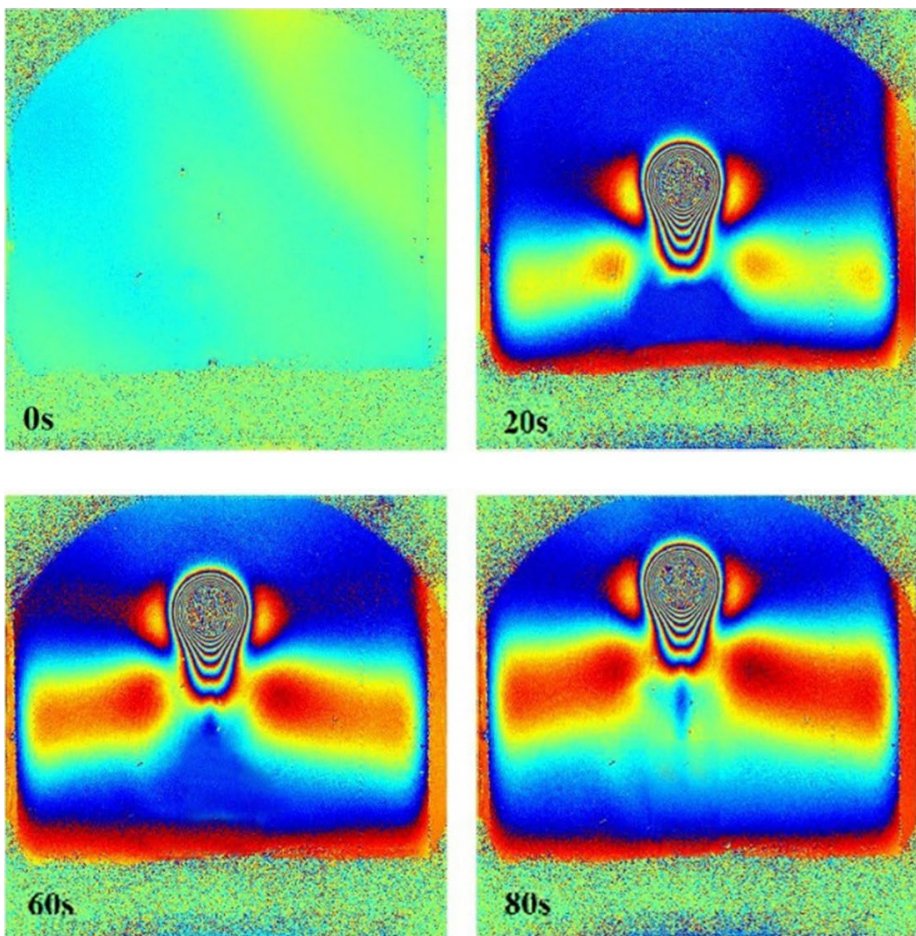

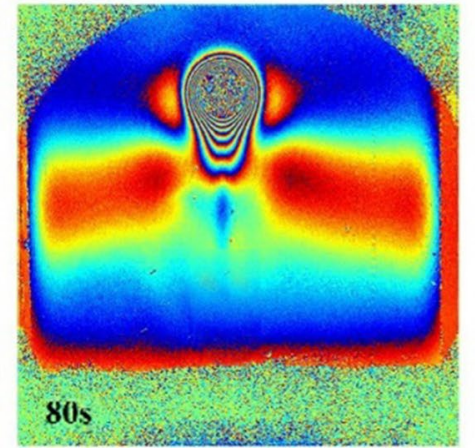

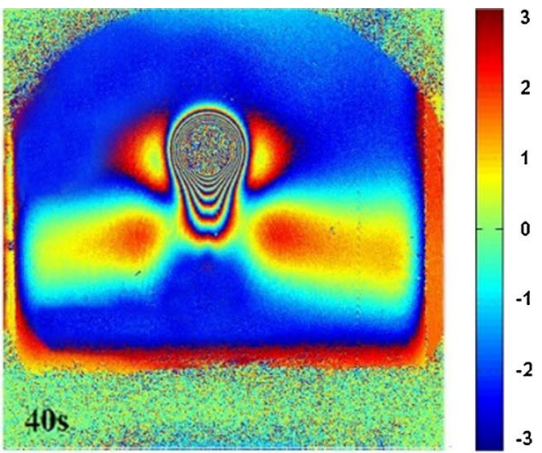

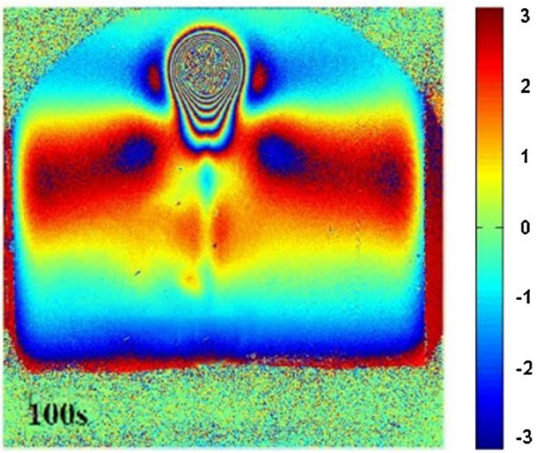

Fig. 8 Reconstructed two-dimensional wrapped phase distributions of the drop migration at different times

Fig. 9 Perturbed temperature distribution and isotherms of the perturbed temperature at $40 \mathrm{~s}$
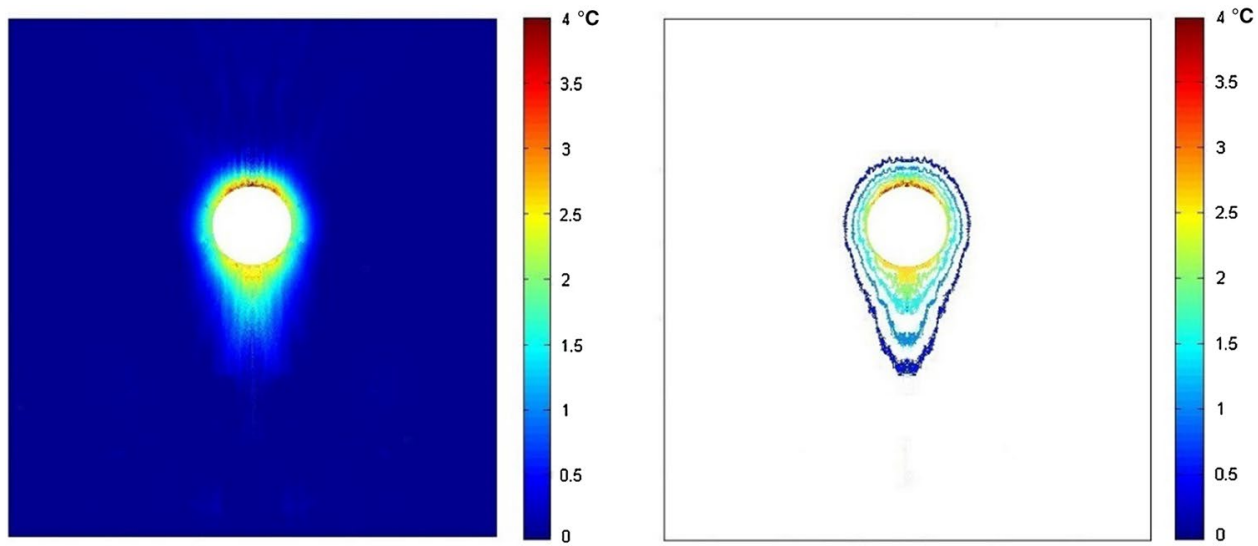

the perturbed temperature distribution and isotherms of the perturbed temperature at $40 \mathrm{~s}$. Figure 10 shows the actual temperature distribution, as well as the isotherms of the actual temperature at $40 \mathrm{~s}$. As shown in Figs. 9 and 10, the drop surface was colder than the continuous phase liquid, and a thermal wake existed behind the drop. The coldness of the drop was due to the predominant convective transport. In the present experiment, the Marangoni number was large, which caused a relatively strong convective transport and weak conduction. The heat was provided mainly by conduction from the continuous phase in order to raise the temperature of the drop, and not by radiation and convection. This mechanism caused the internal and external heat exchange to be relatively weak. As a consequence, a temperature difference between the drop's external and internal existed. Meanwhile, a thermal boundary layer was observed near the drop surface, which maintained the continuity of the heat flux. Therefore, the drop could not immediately warm up, and it was colder than the continuous phase at an equal height. For the above reason, the drop was determined to be cold, and the thermal wake could be clearly seen. The isotherms of the actual temperature, as illustrated in Fig. 10, were neither straight nor vertically symmetrical. The distortion of the isotherms was caused by the convective transport of the heat. 
Fig. 10 Actual temperature distribution and isotherms of the actual temperature at $40 \mathrm{~s}$
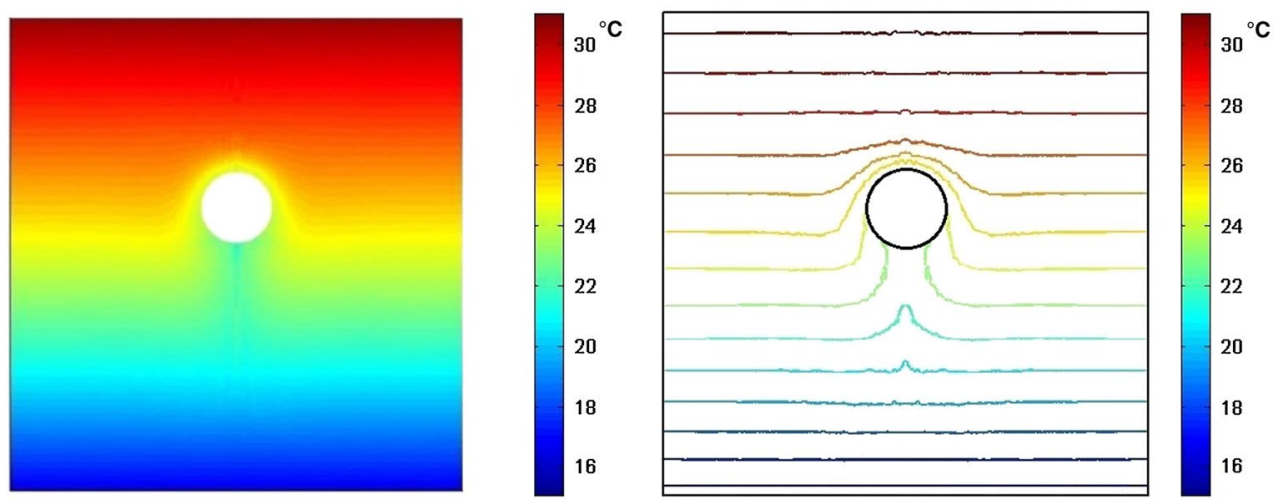

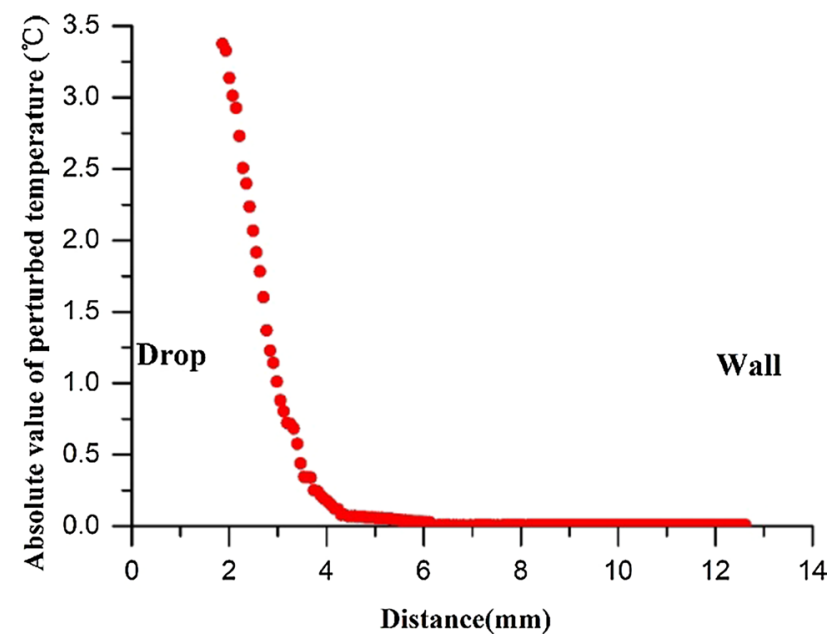

Fig. 11 Perturbed temperature distribution in the horizontal direction

Figure 11 shows the absolute value of the perturbed temperature in the horizontal direction at an angle of $\theta=90^{\circ}$, which was over the centre of the drop. The perturbed temperature at different locations could be obtained by measuring the horizontal direction distance of the drop's centre. It was found that the absolute value of the perturbed temperature at the drop's surface was $3.38{ }^{\circ} \mathrm{C}$. However, this returned to $0.03{ }^{\circ} \mathrm{C}$ when the distance of the drop's surface was $4.04 \mathrm{~mm}$. When the perturbed temperature was equal to $1 \%$ of the perturbed temperature at the drop's surface, the perturbed temperature was considered to have disappeared. Therefore, in the horizontal direction, when the distance from a position to the drop's surface was more than $4.04 \mathrm{~mm}$, the perturbed temperature at such a position was considered to be negligible. As a consequence, outside the perturbed region, the temperature remained unchanged. However, inside the perturbed region, the temperature field was changed. Therefore, if other drops migrated inside the region, they may have been affected.

Figure 12 shows the absolute value of the perturbed temperature in the vertical direction, at angles of $\theta=0^{\circ}$

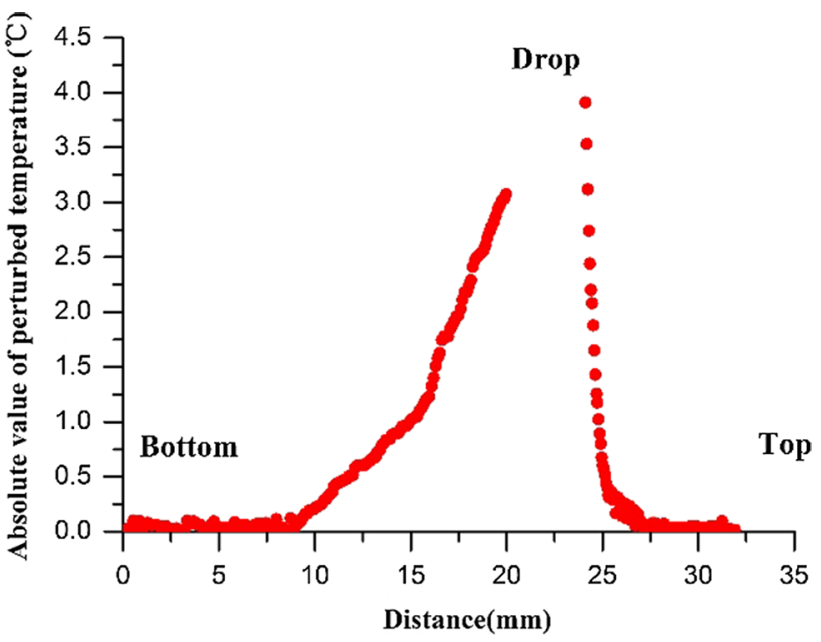

Fig. 12 Perturbed temperature distribution in the vertical direction

and $\theta=180^{\circ}$, which was over the centre of the drop. The perturbed temperature at the different locations could be obtained by measuring the vertical direction distance from the drop's centre. It was found that the absolute value of the perturbed temperature at the forward stagnation point on the surface of the drop was $3.91{ }^{\circ} \mathrm{C}$, and at the rear stagnation point it was $3.07{ }^{\circ} \mathrm{C}$. The absolute value of the perturbed temperature of the forward stagnation point was larger than that of the rear stagnation point on the surface of the drop. This perturbed temperature difference was due to the recirculation in the drop. As a consequence, a temperature gradient which was less than the unperturbed temperature gradient was established between the forward and rear stagnation points on the surface of drop. A lower temperature gradient meant a slower migration velocity, and was the reason why the drop migration velocity of the experiment was slower than that which the YGB linear theory had predicted.

Above the drop, it was found that the absolute value of the perturbed temperature at the forward stagnation point on the drop's surface was $3.91{ }^{\circ} \mathrm{C}$, which returned 
Fig. 13 Results of the 5.95-mm drop
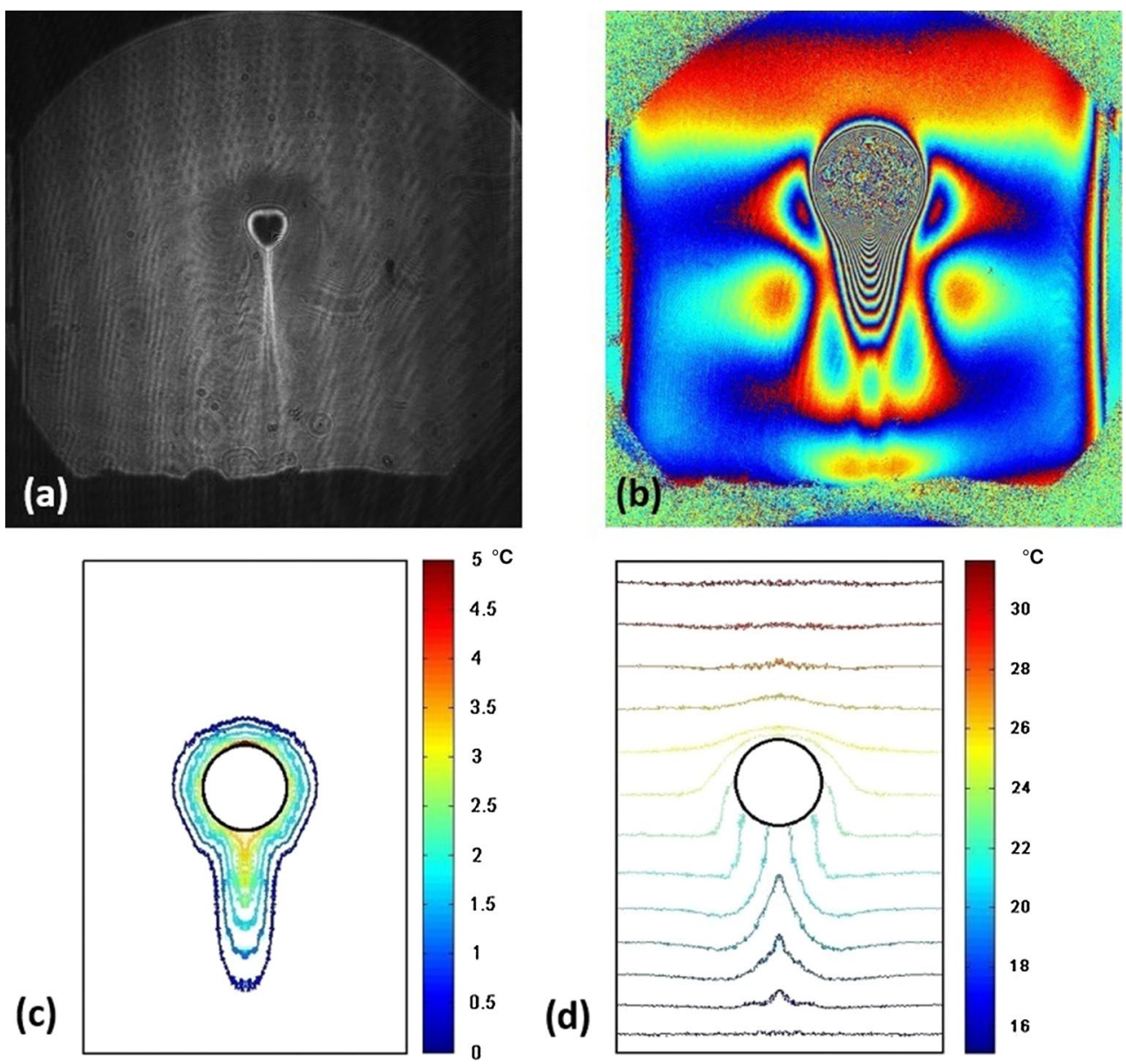

(d)

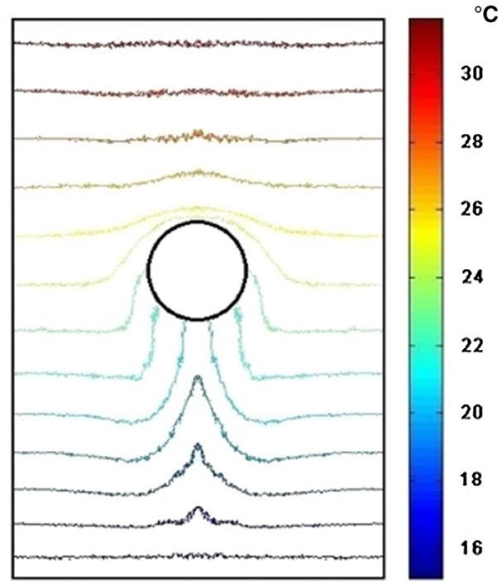

to $0.03{ }^{\circ} \mathrm{C}$ when the distance of the drop surface reached $3.30 \mathrm{~mm}$. Therefore, above the drop and in the vertical direction, when the distance from a position to the drop surface was more than $3.30 \mathrm{~mm}$, the perturbed temperature at such a position could be neglected. On the other side, below the drop, it was found that the absolute value of the perturbed temperature at the rear stagnation point on the drop surface was $3.07{ }^{\circ} \mathrm{C}$, which returned to $0.03{ }^{\circ} \mathrm{C}$ when the distance of drop's surface reached $11.13 \mathrm{~mm}$. Therefore, below the drop and in the vertical direction, when the distance from a certain position to the drop's surface was less than $11.13 \mathrm{~mm}$, the perturbed temperature at this position could not be neglected. A long thermal wake was clearly seen. Consequently, the trailing drop could be affected in the thermal wake region of the leading drop, namely such a region where the perturbed temperature was larger than $1 \%$ of the maximum perturbed temperature.

More explicit details of the 5.95-mm-diameter drop are shown in Fig. 13, whose Reynolds number was 0.430 , Marangoni number was 130, and Capillary number was 0.00434. The hologram is illustrated in Fig. 13a. The wrapped phase map is shown in Fig. 13b, and the isotherms of the perturbed temperature are shown in Fig. 13c. The isotherms of the actual temperature are shown in Fig. 13d. Figure 13 illustrates the fact that the perturbed temperature and the thermal wake region of the 5.95-mm drop were larger than those of the 3.70-mm drop, and the length of the thermal wake region of the 5.95-mm drop was longer than that of the $3.70-\mathrm{mm}$ drop. More results of the perturbed temperature and thermal wake region are summarized in Figs. 14 and 15.

An interfacial tension gradient drove the drop's thermocapillary migration. A larger temperature gradient meant a greater driving force and a faster migration velocity. Therefore, the maximum temperature difference, which was defined as the temperature difference between the forward stagnation point and the rear stagnation point, was found to be an important factor in the drop's thermocapillary migration. In this current study, $\mathrm{T}$ represents the dimensionless interface temperature difference, which was defined as the ratio of maximum interface temperature difference in the experiment to the product of the drop's diameter and temperature gradient in the continuous phase. As shown in Fig. 14, the dimensionless interface temperature difference was a monotonically decreasing function of 


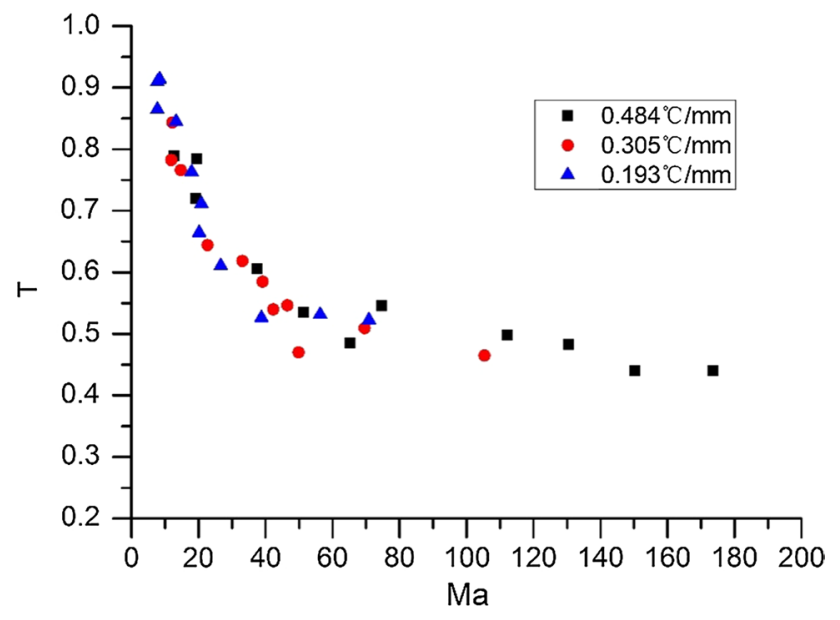

Fig. 14 Dimensionless interface temperature differences plotted against the Marangoni number

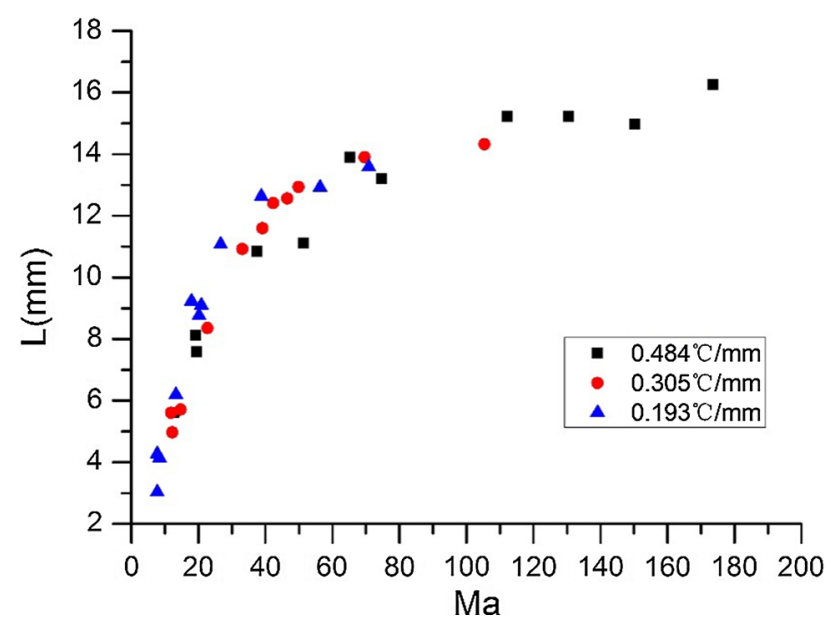

Fig. 15 Length of the thermal wake region plotted against the Marangoni number

the Marangoni number for the three different temperature gradients.

When the Reynolds and Marangoni numbers were infinitesimal, the drop's thermocapillary migration was in accordance with the linear YGB theory. The temperature field around the drop was uniform and straight. The maximum interface temperature difference was the product of the drop's diameter and temperature gradient in the continuous phase, and there was a large temperature difference along the drop's surface. The drop's thermocapillary migration velocity in the experiment was equal to that in the linear YGB theory.

When the Marangoni number was less than 10 , or small and finite, the temperature field around the drop was perturbed by the convective transport of heat. The temperature field became bent and deformed. The dimensionless interface temperature difference was a little less than the product of the drop's diameter and temperature gradient in the continuous phase.

With the further increasing of the Marangoni number, in a range of 10 and 50, the dimensionless interface temperature difference decreased rapidly. The dimensionless interface temperature difference over the drop's surface was reduced by the influence of the enhanced convective transport of heat. The temperature difference between the forward stagnation and the rear stagnation points drove the drop's thermocapillary migration. Therefore, a lower dimensionless interface temperature difference led to less of a driving force, and a slower migration velocity. With the increasing of the Marangoni number, the dimensionless interface temperature difference decreased, which was caused by the enhanced convective transport results in the drop's thermocapillary migration velocity becoming decreased.

When the Marangoni number was larger than 50, the influence of the convective transport of heat further increased. However, the dimensionless interface temperature difference decreased at a slower rate than previously. The dimensionless interface temperature difference decreased from 50 to $40 \%$. The further decreasing of the dimensionless interface temperature difference led to a decrease in the drop's thermocapillary migration velocity.

In earlier space experiments, such as the IML-2 (Balasubramaniam et al. 1996) and LMS (Hadland et al. 1999) experiments, the scaled drop velocity, which was defined as the ratio of the measured velocity to the velocity the drop would have if the convective transport of both momentum and energy were negligible, decreased the Marangoni number. When the Marangoni number was less than 50, the scaled drop velocity had a sharp decrease with increase in the Marangoni number. When Marangoni number was larger than 50, the scaled drop velocity decreased at a slower rate than previously. After comparing the velocity data of earlier space experiments and the temperature data in the present experiment, it could be seen that similarities existed. There was clearly evident that, with an increasing Marangoni number, the dimensionless interface temperature difference decreased, which resulted in the scaled drop velocity also becoming decreased.

In this study, $L$ represents the length of the thermal wake region. As shown in Fig. 15, the length of the thermal wake region increased with the increasing Marangoni numbers for the three different temperature gradients. When the Marangoni number was less than 50, the length of the thermal wake region increased rapidly. A thermal wake appeared in the presence of the convective transport of heat. With further increasing of the Marangoni number (larger than 50), the influence of the convective transport 
of heat further increased. However, the length of the thermal wake region increased at a slower rate than previously. Therefore, with the increasing Marangoni numbers, the length of the thermal wake region increased, and the thermal wake region was extended.

In the thermal wake region, the temperature field became deformed, and the temperature gradient changed. Therefore, if a second drop was inserted behind the leading drop, the thermocapillary migration was found to be different from the migration of the isolated drops. The two drops interacted with each other, and the leading drop could significantly retard the motion of the trailing drop. The temperature field of the two interacting drops will be examined further.

\section{Conclusions}

The thermocapillary migration of drops in a temperature gradient was studied in the present experiment by utilizing digital holographic interferometry. The temperature distribution around a drop, as well as on the interface of a drop, during migration in a vertical temperature gradient were visualized and provided quantitatively for the first time. The perturbed temperature distribution and the actual temperature distribution around the drop during the thermocapillary migration were obtained, and were discussed in detail in this study. The drop was colder than the continuous phase liquid, and a thermal wake existed behind the drop. The dimensionless interface temperature difference was a monotonically decreasing function of the Marangoni number for the three different temperature gradients. When the Marangoni number was increased, the dimensionless interface temperature difference was found to decrease, which was caused by the decrease of the enhanced convective transport results in the drop's thermocapillary migration velocity. With the increasing Marangoni numbers, the length of the thermal wake region increased, and the thermal wake region was extended.

Acknowledgments This research study was funded by the National Natural Science Foundation of China (Grant No. 11372328), by the Strategic Priority Research Program on Space Science, the Chinese Academy of Sciences: SJ-10 Recoverable Scientific Experiment Satellite (Grant Nos. XDA04020405 and XDA04020202-05), and by China Manned Space Engineering program. The authors wish to thank the team led by Professor Jianlin Zhao of Northwestern Polytechnical University for providing assistance in using the digital holographic interferometer.

\section{References}

Akhmetov RG (2004) An asymptotic expansion of the solution to the convective diffusion problem in the trail to the rear of a droplet. Comput Math Math Phys 44(6):1007-1023
Balasubramaniam R, Chai AT (1987) Thermocapillary migration of droplets: an exact solution for small Marangoni numbers. J Colloid Interface Sci 119(2):531-538

Balasubramaniam R, Subramaniam RS (1996) Thermocapillary bubble migration-thermal boundary layers for large Marangoni numbers. Int J Multiph Flow 22(3):593-612

Balasubramaniam R, Subramanian RS (2000) The migration of a drop in a uniform temperature gradient at large Marangoni numbers. Phys Fluids (1994-present) 12(4):733-743

Balasubramaniam R, Lacy CE, Woniak G, Subramanian RS (1996) Thermocapillary migration of bubbles and drops at moderate values of the Marangoni number in reduced gravity. Phys Fluids (1994-present) 8(4):872-880

Duan L, Kang Q, Sun ZW, Hu L, Cui HL, Lin H, Li GP (2008) The real-time Mach-Zehnder interferometer used in space experiment. Microgr Sci Technol 20(2):91-98

Frolovskaya O, Nir A, Lavrenteva OM (2006) Stationary regimes of axisymmetric thermal wake interaction of two buoyant drops at low Reynolds and high Peclet number. Phys Fluids (1994-present) 18(7):072103

Hadland PH, Balasubramaniam R, Wozniak G, Subramanian RS (1999) Thermocapillary migration of bubbles and drops at moderate to large Marangoni number and moderate Reynolds number in reduced gravity. Exp Fluids 26(3):240-248

Hähnel M, Delitzsch V, Eckelmann H (1989) The motion of droplets in a vertical temperature gradient. Phys Fluids A Fluid Dyn (1989-1993) 1(9):1460-1466

Kang Q, Cui HL, Hu L, Duan L (2008) On-board experimental study of bubble thermocapillary migration in a recoverable satellite. Microgr Sci Technol 20(2):67-71

Lavrenteva OM, Nir A (2003) Axisymmetric thermal wake interaction of two drops in a gravity field at low Reynolds and high Peclet numbers. Phys Fluids (1994-present) 15(10):3006-3014

Ma X (1999) Numerical simulation of thermocapillary drop motion with internal circulation. Numer Heat Transfer Part A Appl 35(3):291-309

Polyanin AD (1982) Method for solution of some non-linear boundary value problems of a non-stationary diffusion-controlled (thermal) boundary layer. Int J Heat Mass Transf 25(4):471-485

Polyanin AD (1984) Unsteady-state extraction from a falling droplet with nonlinear dependence of distribution coefficient on concentration. Int J Heat Mass Transf 27(8):1261-1276

Rashidnia N, Balasubramaniam R (1991) Thermocapillary migration of liquid droplets in a temperature gradient in a density matched system. Exp Fluids 11(2-3):167-174

Subramanian RS, Balasubramaniam R (2001) The motion of bubbles and drops in reduced gravity. Cambridge University Press, Cambridge

Wang J, Zhao J, Di J, Rauf A, Hao J (2014) Dynamically measuring unstable reaction-diffusion process by using digital holographic interferometry. Opt Lasers Eng 57:1-5

Wozniak G (1991) On the thermocapillary motion of droplets under reduced gravity. J Colloid Interface Sci 141(1):245-254

Wu ZB (2014) Thermocapillary migration of a droplet with a thermal source at large Reynolds and Marangoni numbers. Int J Heat Mass Transf 75:704-709

Xie JC, Lin H, Han JH, Hu WR (1996) Drop migration of middle Reynolds number in a vertical temperature gradient. Microgr Sci Technol 9(2):95-99

Yarin AL, Liu W, Reneker DH (2002) Motion of droplets along thin fibers with temperature gradient. J Appl Phys 91(7):4751-4760

Young NO, Goldstein JS, Block MJ (1959) The motion of bubbles in a vertical temperature gradient. J Fluid Mech 6(03):350-356

Zhang L, Subramanian RS, Balasubramaniam R (2001) Motion of a drop in a vertical temperature gradient at small Marangoni number-the critical role of inertia. J Fluid Mech 448:197-211 
Zhang L, Duan L, Kang Q (2014) An experimental research on surface oscillation of buoyant-thermocapillary convection in open cylindrical annuli. Acta Mech Sin 30(5):681-686

Zhao JF, Zhang L, Li ZD, Qin WT (2011) Topological structure evolvement of flow and temperature fields in deformable drop
Marangoni migration in microgravity. Int $\mathrm{J}$ Heat Mass Transf 54(21):4655-4663

Zhu P, Zhou B, Duan L, Kang Q (2011) Characteristics of surface oscillation in thermocapillary convection. Exp Thermal Fluid Sci 35(7):1444-1450 